\title{
Optimal method of gold nanoparticle administration in melanoma-bearing mice
}

\author{
TETSUYA KOMATSU $^{1,2}$, KATSUMASA NAKAMURA ${ }^{2}$, YASUHIRO OKUMURA ${ }^{1}$ and KENTA KONISHI ${ }^{2}$ \\ ${ }^{1}$ Department of Radiation Oncology, Tokai University School of Medicine, Isehara, Kanagawa 259-1193; \\ ${ }^{2}$ Department of Radiation Oncology, Hamamatsu University School of Medicine, Hamamatsu, Shizuoka 431-3192, Japan
}

Received January 3, 2017; Accepted April 21, 2017

DOI: $10.3892 /$ etm.2018.5746

\begin{abstract}
The present study assessed different methods of administering gold nanoparticles (GNPs) using different formulations to determine which of the methods achieved optimal radiosensitization. Cells from the B16F10 mouse melanoma cell line were implanted in the femoral area of mice, assigned to one of the eight following groups: i) Control; ii) intravenous (IV) injection of polyethylene glycol (PEG)-binding GNPs (Peg-GNPs) alone; iii) direct intratumoral (IT) injection of Peg-GNPs alone; iv) radiotherapy (RT)-alone; v) Peg-GNP IV + RT; vi) Peg-GNP IT + RT; vii) naked GNP (N-GNPs) $\mathrm{IV}+\mathrm{RT}$; and viii) N-GNP IT + RT. Injection volumes of the Peg-GNPs (particle size, $15 \mathrm{~nm}$; dose, $2.8 \mathrm{mg} / \mathrm{ml}$ ) and N-GNPs (particle size, $15 \mathrm{~nm}$; dose, $200 \mathrm{mg} \mathrm{Au} / \mathrm{cc}$ ) were 0.3 and $0.2 \mathrm{ml}$ per mouse, respectively, for IV and IT. The femoral area was irradiated with a single dose of $10 \mathrm{~Gy}$. To evaluate the effects of GNPs, the current study measured the changes in the tumor volume ratio to the initial tumor volume over time and observed the survival rate. Administration of GNPs with RT did not improve the suppression of tumor growth or survival to a statistically significant extent. The administration of Peg-GNPs alone indicated a slight tumor suppressing effect at the early stage. The current study was not able to confirm the radiosensitization effect of GNPs in melanoma-bearing mice with tumors that were large in comparison to previous studies. Further research is required to validate the radiosensitizing effect on large tumors.
\end{abstract}

\section{Introduction}

In 2004, the effectiveness of gold nanoparticles (GNPs) as a radiosensitizer in vivo was first demonstrated by Hainfeld et al (1). Over the next decade, numerous studies

Correspondence to: Dr Tetsuya Komatsu, Department of Radiation Oncology, Hamamatsu University School of Medicine, 1-20-1 Handayama, Hamamatsu, Shizuoka 431-3192, Japan

E-mail:komte@hama-med.ac.jp

Key words: gold nanoparticles, melanoma, radiosensitizer, mouse, survival were performed to investigate the optimal treatment parameters (2-6). The mechanism underlying the radiosensitizing effect of GNPs is not fully understood and a number of mechanisms have been suggested, including increased photoelectric photon absorption with a high-Z material, or an insult to the tumor blood vessels via an anti-angiogenic effect $(3,7,8)$. In a previous study, GNPs were administered via intravenous (IV) injection or direct intratumoral (IT) injection (5); however, to the best of our knowledge, no studies have been performed to compare these two routes of administration.

When GNPs of an appropriate size are intravenously administered, particles accumulate in the tumor via the enhanced permeability and retention effect (9). However, the particles are primarily incorporated into other organs, and only a limited amount reaches the tumor (1). Intratumoral injection of particles increases the density of GNPs within the tumor, which may increase the treatment effect; however, this method is disadvantageous as it results in heterogeneous distribution (10).

The degree of GNP-induced radiosensitization is also reported to differ among various cell types $(11,12)$. Malignant melanoma is radiation-resistant and typically develops on the skin surface. Therefore, low-energy X-ray treatment is possible and malignant melanoma may allow a good indication for the successful clinical application of this treatment method. Previously, two studies on the radiosensitization effects of GNPs in malignant melanoma bearing mice have been reported $(13,14)$. Although the experiments of the two studies were performed under similar conditions, their results were inconsistent regarding the radiosensitizing effect, with one study indicating enough radiosensitizing effect of GNP, but the other study unable to replicate the same results. For further understanding, the present study performed experiments under treatment conditions that differed in tumor size from the two previous studies.

Recently, the modification of GNPs with polyethylene glycol (PEG)-binding (PEGylation) has been reported to achieve a favorable treatment effect (15-18). As a foreign body, GNPs are rapidly incorporated and removed by the reticuloendothelial system; PEGylation inhibits this reaction (18) and may lead to a tumor-specific treatment effect. The influence of PEGylation was not evaluated in the previous studies using 
malignant melanoma bearing mice $(13,14)$. Therefore, it was included in the present investigation.

\section{Materials and methods}

Animal model. A total of 50, 6-week-old nude mice (body weight $18.3 \pm 1.5 \mathrm{~g}$ ) BALB/C-nu/nu (female), were purchased from CLEA Japan Inc. (Tokyo, Japan). Housing conditions for all mice were as follows: Temperature $20-26^{\circ} \mathrm{C}$ and humidity 40-60\%. A 12-h light/dark cycle was used. Food and water were provided ad libitum). Experiments were performed at Tokai University Animal Experiment Center (Kanagawa, Japan) in accordance with the Tokai University guidelines and the experimental protocol was approved by the Institutional Animal Care and Use Committee (Department of Radiation Oncology, Tokai University, Kanagawa, Japan) prior to the start of the study. The murine malignant melanoma cell line, B16F10 (American Type Culture Collection, Manassas, VA, USA), was cultured in Dulbecco's modified Eagle medium (DMEM; Wako Pure Chemical Industries, Ltd., Osaka, Japan) at $37^{\circ} \mathrm{C}$ in a $5 \% \mathrm{CO}_{2}$ atmosphere. The culture media contained penicillin $(50 \mathrm{IU} / \mathrm{ml})$ and streptomycin $(50 \mu \mathrm{g} / \mathrm{ml})$ and was supplemented with 10\% fetal calf serum (Sigma-Aldrich; Merck KGaA, Darmstadt, Germany). B16F10 cells were cultured for 4 days at a density of $1 \times 10^{5}$ cells in $7 \mathrm{ml}$ DMEM in a $100-\mathrm{mm}$ culture dish (Eppendorf, Hamburg, Germany) at $37^{\circ} \mathrm{C}$ in a $5 \% \mathrm{CO}_{2}$ atmosphere. Following two washes with phosphate-buffered saline (PBS) for $\sim 5 \mathrm{~min}$, the B16F10 cells were harvested with $0.25 \mathrm{w} / \mathrm{v} \%$ trypsin-1 mmol/l EDTA-4Na solution with phenol red (Wako Pure Chemical Industries, Ltd.). Harvested B16F10 cells were suspended in PBS, and the cell density was adjusted $\left(2 \times 10^{6}\right.$ cells $\left./ 100 \mu \mathrm{l}\right)$. A total of $2 \times 10^{5}$ cells were subcutaneously inoculated in the femoral region of all mice.

Treatment groups. Two types of GNPs were used: PEGylated GNPs (Peg-GNPs) and naked GNPs (N-GNPs). In total, $2.8 \mathrm{mg} / \mathrm{ml}$ methyl-terminated 5000 PEG-coated GNPs (diameter, $5 \mathrm{~nm}$; Sigma-Aldrich; Merck KGaA) were used as Peg-GNPs and GNPs at a concentration of $200 \mathrm{mg} \mathrm{Au} / \mathrm{cc}$ (diameter, $15 \mathrm{~nm}$; Nanoprobes Inc., Yaphank, NY, USA) were used as N-GNPs.

Eight treatment groups were established, as follows: i) Control ( $n=10)$; ii) Peg-GNP IV-alone ( $n=5)$; iii) Peg-GNP IT-alone (n=5); iv) radiotherapy (RT)-alone ( $\mathrm{n}=10)$; v) Peg-GNP $\mathrm{IV}+\mathrm{RT}(\mathrm{n}=5)$; vi) Peg-GNP IT + RT (n=5); vii) N-GNP $\mathrm{IV}+\mathrm{RT}(\mathrm{n}=5)$ and viii) N-GNP IT + RT $(\mathrm{n}=5)$. Each group consisted of 5 mice for each experiment. Mice were randomly allocated so as to prevent variation in the mean tumor volume among the groups. The experiment was performed in two stages: The first stage included groups i-vi; the second stage included groups i, iv, vii and viii. Accordingly, Groups i and iv consisted of 10 mice. Treatment was initiated when the tumor diameter reached $\sim 1 \mathrm{~cm}$ at 12-19 days following transplantation (Fig. 1).

To ensure that the growth ability of the grafted tumors was uniform, 19 animals with a tumor volume of $\leq 200$ or $\geq 1,300 \mathrm{~mm}^{3}$ at $12-19$ days following cell transplantation were excluded from the analysis. Finally, the number of mice for the analysis is as follows: i) Control ( $n=7)$; ii) Peg-GNP IV-alone ( $\mathrm{n}=3$ ); iii) Peg-GNP IT-alone ( $\mathrm{n}=3$ ); iv) radiotherapy (RT)-alone (n=5); v) Peg-GNP IV + RT (n=3); vi) Peg-GNP
IT + RT (n=3); vii) N-GNP IV + RT ( $\mathrm{n}=4)$ and viii) N-GNP IT + RT (n=3).

GNPs were injected via the tail vein or directly into the tumor using a $27-\mathrm{G}$ needle attached to a $1-\mathrm{ml}$ syringe (Terumo Corp., Tokyo, Japan). Injection volumes (both IV and IT) of Peg-GNPs and N-GNPs were 0.3 and $0.2 \mathrm{ml}$ per mouse, respectively. With respect to IV of N-GNPs, the dose and concentrations were almost equal to that of Hainfeld et al's research (1). As the concentration of PEG-GNPs is lower than that of N-GNPs, PEGylation was expected to lead to a much higher accumulation of GNPs in the tumor when compared with N-GNPs.

X-ray irradiation. A MBR-1520R-3 (Hitachi Medical Corp., Tokyo, Japan) device was used to perform X-ray irradiation (settings, $150 \mathrm{kv}$ and $20 \mathrm{~mA}$, which are commonly used in such experiments.). A filter (0.5-mm-thick aluminum + 0.1-mm-thick copper) was used, and the distance between the radiation source and the skin was set at $55 \mathrm{~cm}$. With the exception of the femoral region on the affected side, the mouse body was protected with a 2 -cm thick block prepared with a low-melting-point lead alloy (cerrobend block). Irradiation was performed without anesthesia or sedation using a retainer prepared with a $50-\mathrm{ml}$ conical tube. A dose of $10 \mathrm{~Gy}$ was delivered in a single fraction.

Tumor assessment. Following treatment, the major and short axes of the tumor were measured over time, and the tumor volume was calculated using the following approximation formula: Tumor volume $=\left(\right.$ major axis $\mathrm{x}$ short axis $\left.{ }^{2}\right) / 2\left(\mathrm{~mm}^{3}\right)$. Tumor diameter was measured on alternate days, three times per week for three weeks, and the tumor volume ratio at each measurement time-point to the tumor volume at the time of treatment initiation was calculated as the tumor volume ratio (TVR) using the following formula: TVR = (tumor volume at the measurement time point)/(tumor volume immediately prior to treatment initiation). TVR was used as an index of the tumor growth rate. The time-course of the tumor volume ratio was evaluated, and compared among the groups.

Statistical analysis. Statistical analyses were performed using SPSS software (version 23; IBM Corp., Armonk, NY, USA). Mean values were compared using Student's t-test (two-sided). Survival analysis was performed using the Kaplan-Meier method, and the survival rate was compared using the log-rank test. The data is presented as the mean \pm standard deviation. $\mathrm{P}<0.05$ was considered to indicate a statistically significant difference.

\section{Results}

Suppression of tumor growth. Fig. 2 presents the mean TVR values (Fig. 2A) and tumor volume (Fig. 2B) of each group over the time-course of the experiment. The TVR value was highest in the control group, followed by the groups that were treated with the drug alone without irradiation. The mean values of the four groups that were treated with a combination of the drug and irradiation were similar. Significant differences were observed between the RT groups (RT-alone, RT + Peg-GNP IV, RT + Peg-GNP IT and RT + N-GNP IT) 


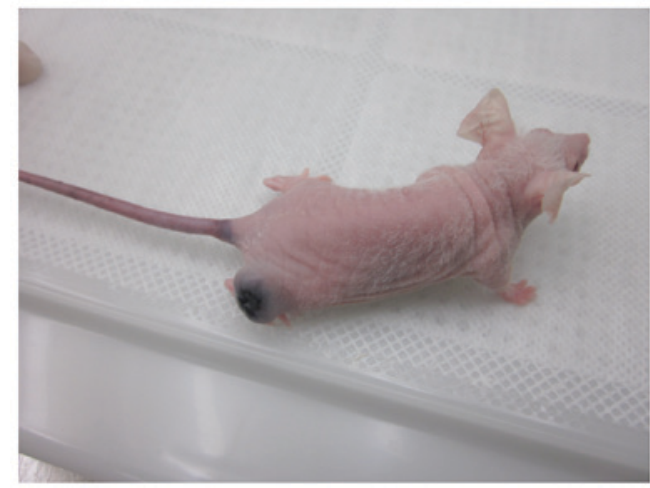

Figure 1. Melanoma-bearing mouse prior to treatment.
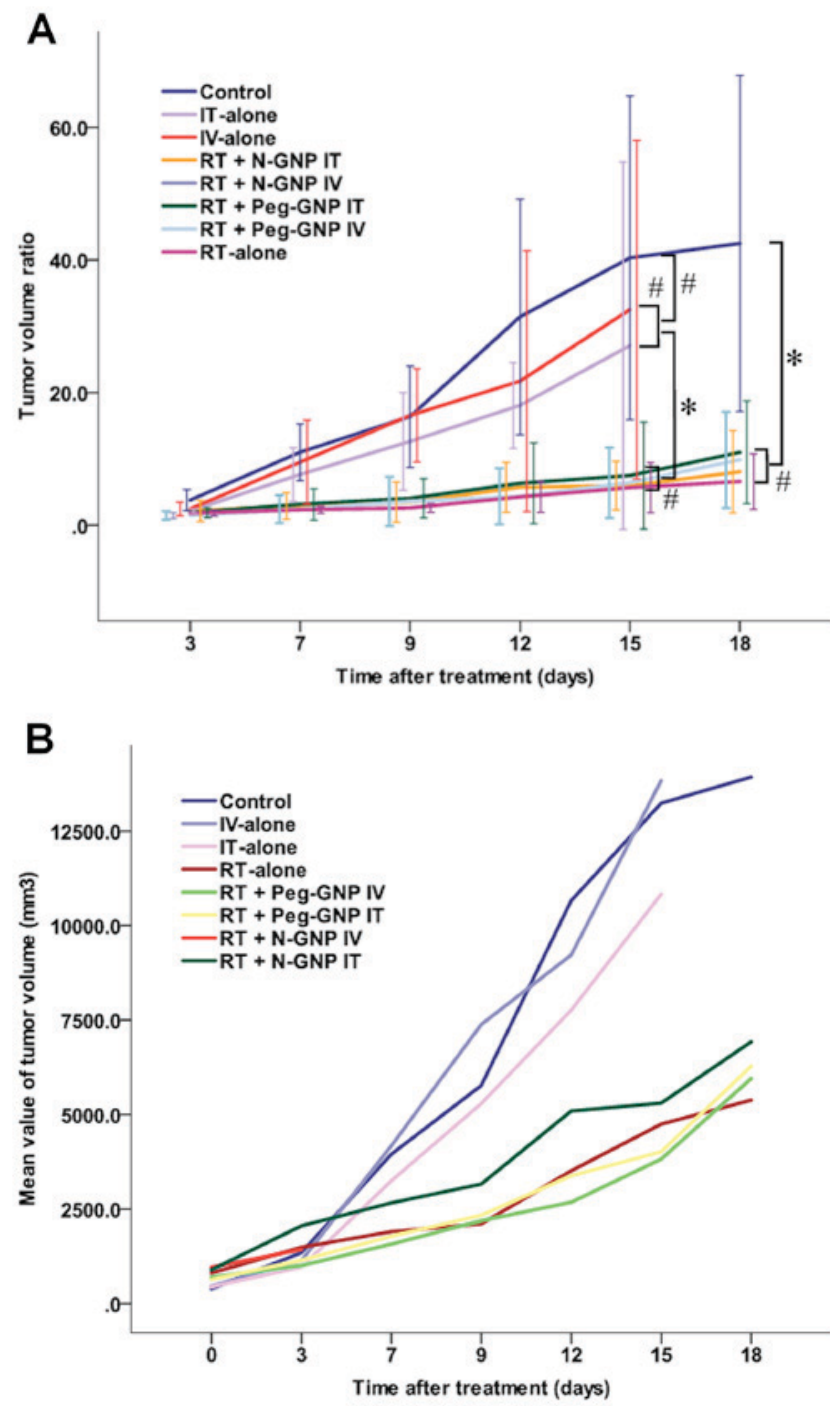

Figure 2. Tumor growth following treatment. (A) Mean tumor volume ratio values of each group over the time-course of the experiment. The tumor volume ratio indicates the ratio of the tumor volume at each time-point to the initial tumor volume. The error bars indicate the $95 \%$ confidence intervals Among the RT groups (RT-alone, RT + Peg-GNP IV, RT + Peg-GNP IT and RT + N-GNP IT), no statistically significant differences were observed. (B) Mean tumor volume of each group over the time-course of the experiment. ${ }^{~} \mathrm{P}<0.05 \mathrm{RT}$ groups vs. control or Peg-GNP alone, ${ }^{\sharp} \mathrm{P}>0.05 \mathrm{IV}$-alone vs. IT alone, control vs. Peg-GNP alone. Data are presented as mean \pm standard deviation. RT, radiotherapy; GNP, gold nanoparticles; N-GNP, naked GNP Peg-GNP, polyethylene glycol-binding GNP; IV, intravenous injection; IT, intratumoral injection.

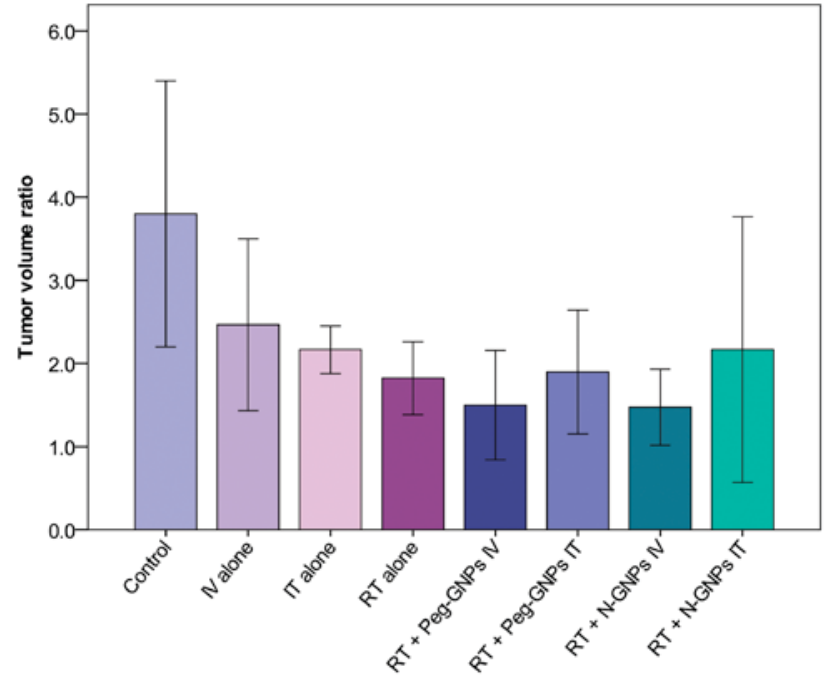

Figure 3. Range of tumor volume ratio in each group 3 days after treatment. The RT + IV groups (RT + Peg-GNPs IV and RT + N-GNPs IV) exhibited a slight decrease in value when compared with the radiation-alone group. RT, radiotherapy; GNP, gold nanoparticles; N-GNP, naked GNP; Peg-GNP, polyethylene glycol-binding GNP; IV, intravenous injection; IT, intratumoral injection.

and the control groups, and the RT groups and the drug alone groups (Fig. 2A).

Fig. 3 presents a comparison of the TVR among the treatment groups on day 3 after the initiation of treatment. A tumor growth-inhibitory effect was observed in all of the treatment groups when compared with the control group. The mean values of the groups in which IV-injected GNPs were administered in combination with RT compared with the RT-alone group and the Peg-GNP and N-GNP groups demonstrated a slight radiosensitization effect, observed as a decrease in tumor volume.

Table I presents the results of the analysis of significance for inter-group differences in the TVR on days 3 and 15. As 3 and 1 of the 4 mice in the N-GNP IV + RT group succumbed to their symptoms on days 5 and 6 after the initiation of treatment, respectively, this group was not included in the evaluations after day 7. In this group, one mouse was initially excluded due to a large tumor volume $\left(2,176 \mathrm{~mm}^{3}\right) 12$ days following cell transplantation. Tumor volume of other mice in this group were $600,726,1,224,1,296 \mathrm{~mm}^{3}$. The anti-tumor effect of the drug alone was evaluated by comparing the control group to the Peg-GNP IV-alone and Peg-GNP IT-alone groups. A significant tumor-inhibitory effect was observed on day 3 in the IT group $(\mathrm{P}=0.047)$; however, the difference on day 15 was no longer significant. The radiosensitization effects with GNPs and the influence of the administration methods were evaluated in the Peg-GNP IT + RT, Peg-GNP IT + RT, $\mathrm{N}$-GNP IV + RT and N-GNP IT + RT groups vs. the RT-alone group. On day 3 , the mean TVR was reduced in the Peg-GNP IT + RT and N-GNP IV + RT groups in comparison with the RT-alone group (1.8 vs. 1.5 and 1.8 vs. 1.4, respectively); however the differences were not statistically significant $(\mathrm{P}=0.177$ and $\mathrm{P}=0.129$, respectively). Thereafter, the growth rate increased in the Peg-GNP IV+ RT group and the values were reversed on day 15. The Peg-GNP IT-alone, Peg-GNP IT + RT and N-GNP IT + RT Peg-GNP IV-alone groups were 
Table I. Comparison of the different treatment groups at 3 and 15 days.

\begin{tabular}{|c|c|c|c|c|}
\hline \multirow[b]{2}{*}{ Group } & \multicolumn{2}{|c|}{3 days } & \multicolumn{2}{|c|}{15 days } \\
\hline & Mean value & P-value & Mean value & $\mathrm{P}$-value \\
\hline Control vs. RT alone & & 0.024 & & $0.014^{\mathrm{a}}$ \\
\hline Control & 3.8 & & 40.3 & \\
\hline RT-alone & 1.8 & & 5.6 & \\
\hline Control vs. Peg-GNP IV-alone & & 0.091 & & 0.605 \\
\hline Control & 3.8 & & 40.3 & \\
\hline Peg-GNP IV-alone & 2.4 & & 32.5 & \\
\hline Control vs. Peg-GNP IT-alone & & $0.047^{\mathrm{a}}$ & & 0.392 \\
\hline Control & 3.8 & & 40.3 & \\
\hline Peg-GNP IT-alone & 2.1 & & 27 & \\
\hline RT-alone vs. Peg-GNP IV + RT & & 0.177 & & 0.68 \\
\hline RT-alone & 1.8 & & 5.6 & \\
\hline Peg-GNP IV + RT & 1.5 & & 6.4 & \\
\hline RT-alone vs. Peg-GNP IT + RT & & 0.745 & & 0.424 \\
\hline RT-alone & 1.8 & & 5.6 & \\
\hline Peg-GNP IT + RT & 1.9 & & 7.5 & \\
\hline RT-alone vs. N-GNP IV + RT & & 0.129 & & \\
\hline RT-alone & 1.8 & & & \\
\hline N-GNP IV + RT & 1.4 & & & \\
\hline RT-alone vs. N-GNP IT + RT & & 0.375 & & 0.859 \\
\hline RT-alone & 1.8 & & 5.6 & \\
\hline N-GNP IT + RT & 2.1 & & 5.9 & \\
\hline Peg-GNP IV-alone vs. Peg-GNP IT-alone & & 0.295 & & 0.568 \\
\hline Peg-GNP IV-alone & 2.4 & & 32.5 & \\
\hline Peg-GNP IT-alone & 2.1 & & 27 & \\
\hline Peg-GNP IV + RT vs. Peg-GNP IT + RT & & 0.158 & & \\
\hline Peg-GNP IV + RT & 1.5 & & 6.4 & 0.659 \\
\hline Peg-GNP IT + RT & 1.9 & & 7.5 & \\
\hline N-GNP IV + RT vs. N-GNP IT + RT & & 0.108 & & \\
\hline N-GNP IV + RT & 1.4 & & & \\
\hline N-GNP IT + RT & 2.1 & & & \\
\hline Peg-GNP IV + RT vs. N-GNP IV + RT & & 0.911 & & \\
\hline Peg-GNP IV + RT & 1.5 & & & \\
\hline N-GNP IV + RT & 1.4 & & & \\
\hline Peg-GNP IT + RT vs. N-GNP IT + RT & & 0.551 & & 0.497 \\
\hline Peg-GNP IT + RT & 1.9 & & 7.5 & \\
\hline N-GNP IT + RT & 2.1 & & 5.9 & \\
\hline
\end{tabular}

${ }^{\mathrm{a}} \mathrm{P}<0.05$. As 3 and 1 of the 4 mice in the N-GNP IV + RT group succumbed on days 5 and 6 after the initiation of treatment, respectively, this group was not included in the evaluations after day 7. RT, radiotherapy; GNP, gold nanoparticles; Peg-GNP, polyethylene glycol-binding GNP; IV, intravenous injection; IT, intratumoral injection; N-GNP, naked GNP.

compared with the Peg-GNP IT + RT group to assess the differences in the effects that occurred due to the variation in the administration route. No significant differences due to the variation in the administration route were observed in either the drug-alone group (Peg-GNP IT-alone), or in the irradiation-combined groups, (Peg-GNP IT + RT or N-GNP IT + RT). In the N-GNP IV + RT and N-GNP IT + RT groups, the difference due to variations in the drug type with the same route of administration was assessed. The drug type was not observed to have an impact on the treatment effect.
Survival time. Fig. 4 presents the survival time of the groups treated with Peg-GNPs (Fig. 4A) and N-GNPs (Fig. 4B) employing each administration method. The addition of GNPs did not improve the survival time. The results of the inter-group comparisons, which were performed using the log-rank test, were as follows: i) A significant difference was observed between the control and RT-alone groups $(\mathrm{P}=0.023)$, and the survival time was longer in the RT-alone group. ii) Combined treatment with GNPs and radiotherapy led to a significantly more favorable survival time in comparison 
Table II. Summary of the previous studies on the radiosensitization effect in B16F10 bearing mice.

Studies

\begin{tabular}{lccc}
\cline { 2 - 4 } Parameter & $\begin{array}{c}\text { Chang et al }, \\
2008(13)\end{array}$ & $\begin{array}{c}\text { Mousavie Anijdan } \text { et al, } \\
2013(14)\end{array}$ & $\begin{array}{c}\text { Present study } \\
\text { (Naked GNP cases) }\end{array}$ \\
\hline Diameter of GNP, $\mathrm{nm}$ & 13 & 50 & 15 \\
Concentration and amount of GNP & $200(\mathrm{nM}) \times 0.2(\mathrm{ml})$ & $5(\mathrm{mg} / \mathrm{ml}) \times 0.1-0.2(\mathrm{ml})$ & $200(\mathrm{mg} / \mathrm{ml}) \times 0.2(\mathrm{ml})$ \\
Administration route & Intravenous & Intratumoral & Intravenous and Intratumoral \\
Tumor volume, $\mathrm{mm}^{3}$ & $50-90$ & $400-600$ & $200-1300$ \\
Radiation energy & $6 \mathrm{MeV}$ electrons & 6 and $18 \mathrm{MV}$ X-ray & $150 \mathrm{kv} \mathrm{X-ray}$ \\
Radiation dose, Gy/1 fraction & 25 & 20 & 10 \\
Tumor growth & Suppressed & Partially suppressed & Not suppressed \\
Statistical survival benefit & Yes & No & No \\
Effect of GNP alone & No & No & Not performed but partially \\
& & & observed in Peg-GNP \\
\hline
\end{tabular}

GNP, gold nanoparticles; Peg-GNP, polyethylene glycol-binding GNP.

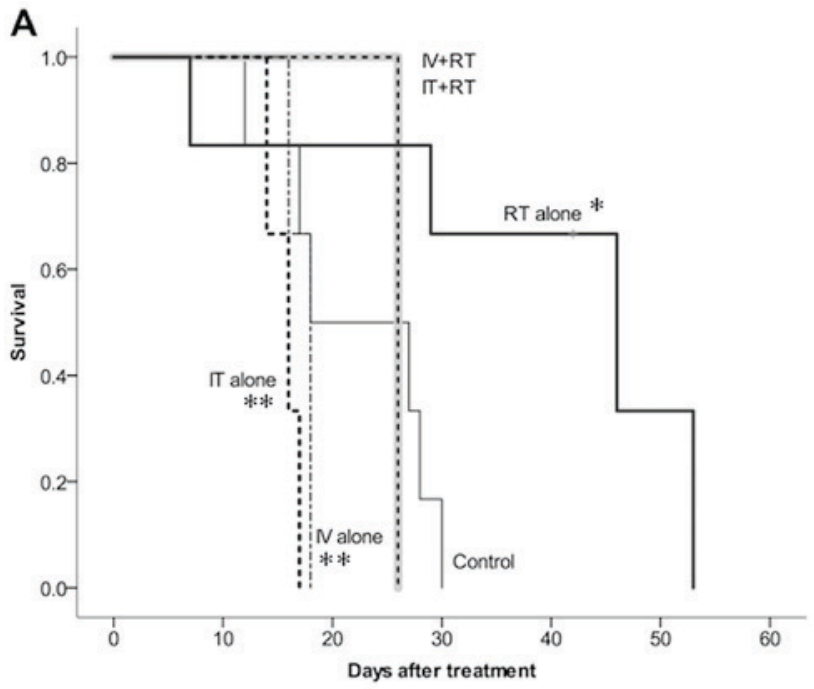

B

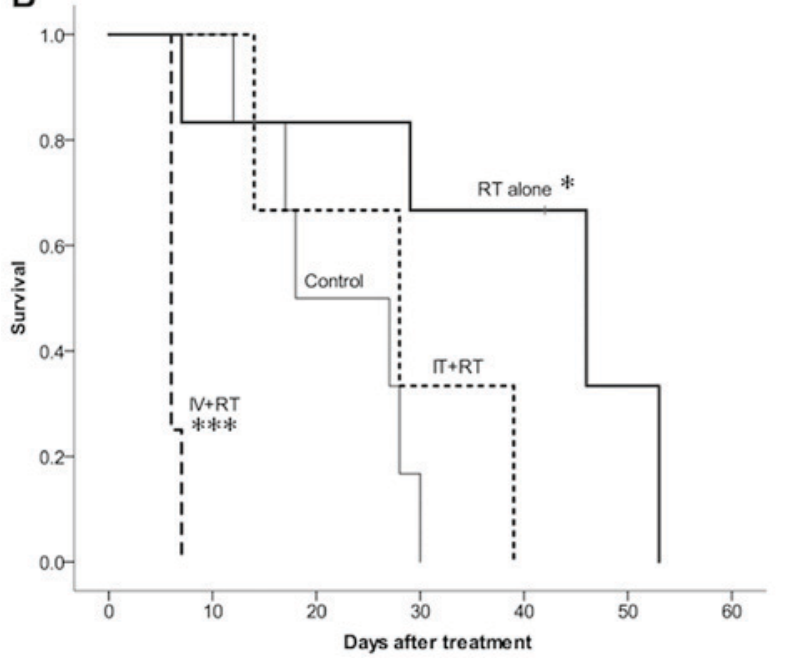

Figure 4. Survival rate following treatment. Survival rate of the (A) polyethylene glycol-binding GNP group and (B) the naked GNP group. " $\mathrm{P}<0.05$ control vs. RT alone, ${ }^{* *} \mathrm{P}<0.05$ GNP-alone vs RT + GNP, ${ }^{* * *} \mathrm{P}<0.05 \mathrm{~N}-\mathrm{GNP}$ IV + RT vs. RT alone. RT, radiotherapy; GNP, gold nanoparticles; IV, intravenous injection; IT, intratumoral injection. to the GNP-alone groups (Peg-GNP IT-alone vs. Peg-GNP IT + RT, P=0.025; Peg-GNP IV-alone vs. Peg-GNP IV + RT, $\mathrm{P}=0.030$ ). iii) Combined treatment with GNPs and irradiation did not lead to a significant improvement in survival time when compared with the RT-alone group (RT-alone vs. Peg-GNP $\mathrm{IV}+\mathrm{RT}, \mathrm{P}=0.070$; RT-alone vs. Peg-GNP IT + RT, $\mathrm{P}=0.070$; RT-alone vs. N-GNP IT + RT, P=0.094). The survival time was significantly reduced in the N-GNPs IV + RT group (RT-alone vs. N-GNPs IV + RT, P=0.004).

\section{Discussion}

GNP-induced radiosensitization in vivo was initially reported by Hainfeld et al in 2004 (1). Subsequent studies have clarified that the degree of radiosensitization is influenced by radiation energy, drug concentration, particle size and cell type $(2,6,11,19)$. Since malignant melanoma is a superficial radiation-resistant tumor, it may be a good indicator for the success of this treatment method; however, very few in vivo studies have been performed to investigate the effects in melanoma.

In 2008, Chang et al (13) reported GNP-induced radiosensitization with electron beams in mice that were implanted with cells from the B16F10 melanoma cell line (13). Previous studies clarified that the degree of radiosensitization is proportionate to the concentration of GNPs (8); however the dose of the drug in the present study was 40- to 50-fold higher than in the studies of Chang et al (13) or Mousavie Anijdan et al (14). In addition to the drug concentration, there were differences in other experimental conditions, including the radiation energy, radiation dose and the timing of treatment initiation. In a previous study, treatment was initiated at a high dose (single dose of electron beam treatment: 25 Gy) 7 days after transplantation, and the tumor volume at this time-point was $50-90 \mathrm{~mm}^{3}$, which was markedly smaller than that at the start of the current experiment (13).

In 2013, Mousavie Anijdan et al (14) performed an experiment under similar conditions, using megavoltage X-rays, but only partial radiosensitization was observed (14). In the present experiment, the mean TVR value 3 days after intravenous 
injection was slightly lower in the IV-combined (Peg-GNPs and N-GNPs) groups than in the RT-alone group, which suggests the presence of slight radiosensitization; however the difference was not statistically significant. The results reported by Mousavie Anijdan et al (14) were similar to the findings of the present study in that GNP-induced radiosensitization was observed in the first month, but overall, it did not lead to a significant difference (14).

Table II compares the results of the present experiment with those of previous studies using malignant melanoma-implanted mice. However, whether the ineffectiveness of GNPs as a radiosensitizer observed in the current study was caused by the physical aspects (radiation dose or energy), or the biological aspects of the experiments, remains to be elucidated.

Mousavie Anijdan et al (14) stated that the tumor volume may markedly influence the radiosensitization effect.

There were also differences in a number of other conditions; however, the findings of the present study did not contradict previous conclusions. In highly malignant tumors, such as melanoma, the tumor volume may influence the radiosensitization effect. Therefore, the association between the tumor size and the radiosensitizing effect should be evaluated in future studies.

Regarding the effect of GNPs alone, Peg-GNPs were only observed to have an antitumor effect in the early period after treatment initiation. Treatment with GNPs alone was not reported to have an effect by Mousavie Anijdan et al (14); however, Chang et al (13) reported a slight increase in the apoptosis activity level in comparison with the control group. A number of studies have reported that GNPs themselves decrease clonogenic survival, increase apoptosis and induce DNA damage (20-22). Furthermore, Butterworth et al (23) demonstrated that these cytotoxic effects were cell-type specific. The results of the present study may reflect these toxic effects. As the present experiment included mice with tumors that were large in comparison to those of previous studies, a generous amount of GNPs at a commercially-available dose was administered. This may have had a negative effect on survival. Whether the decreased survival was associated with the impact of the initial tumor size at the time of treatment or the toxicity of GNPs itself was not determined. These issues should be further studied with a view toward the clinical application of this treatment in the future.

In conclusion, the current study could not confirm the radiosensitization effect of GNPs in melanoma bearing mice with tumors that were larger in size in comparison to previous experiments. However, Peg-GNP-alone demonstrated a slight tumor suppression effect in the early stage of treatment. Further research is required to validate the radiosensitizing effect on large tumors.

\section{Acknowledgements}

The current study was supported by the Japan Society for the Promotion of Sciences, Tokyo, Japan (grant no. JP25461928).

\section{References}

1. Hainfeld JF, Slatkin DN and Smilowitz HM: The use of gold nanoparticles to enhance radiotherapy in mice. Phys Med Biol 49: N309-N315, 2004.
2. Hainfeld JF, Dilmanian FA, Slatkin DN and Smilowitz HM: Radiotherapy enhancement with gold nanoparticles. J Pharm Pharmacol 60: 977-985, 2008.

3. Mesbahi A: A review on gold nanoparticles radiosensitization effect in radiation therapy of cancer. Rep Pract Oncol Radiother 15: 176-180, 2010.

4. Jain S, Hirst DG and O'Sullivan JM: Gold nanoparticles as novel agents for cancer therapy. Br J Radiol 85: 101-113, 2012.

5. Cooper DR, Bekah D and Nadeau JL: Gold nanoparticles and their alternatives for radiation therapy enhancement. Front Chem 2: 86, 2014.

6. Babaei M and Ganjalikhani M: The potential effectiveness of nanoparticles as radio sensitizers for radiotherapy. Bioimpacts 4: 15-20, 2014.

7. Rahman WN, Bishara N, Ackerly T, He CF, Jackson P, Wong C, Davidson R and Geso M: Enhancement of radiation effects by gold nanoparticles for superficial radiation therapy. Nanomedicine 5: 136-142, 2009.

8. Yamada M, Foote M and Prow TW: Therapeutic gold, silver and platinum nanoparticles. Wiley Interdiscip Rev Nanomed Nanobiotechnol 7: 428-445, 2015.

9. Wang AZ, Langer R and Farokhzad OC: Nanoparticle delivery of cancer drugs. Annu Rev Med 63: 185-198, 2012.

10. Herold DM, Das IJ, Stobbe CC, Iyer RV and Chapman JD: Gold microspheres: A selective technique for producing biologically effective dose enhancement. Int J Radiat Biol 76: 1357-1364, 2000.

11. Coulter JA, Jain S, Butterworth KT, Taggart LE, Dickson GR, McMahon SJ, Hyland WB, Muir MF, Trainor C, Hounsell AR, et al: Cell type-dependent uptake, localization, and cytotoxicity of $1.9 \mathrm{~nm}$ gold nanoparticles. Int J Nanomedicine 7: 2673-2685, 2012

12. Jain S, Coulter JA, Hounsell AR, Butterworth KT, McMahon SJ, Hyland WB, Muir MF, Dickson GR, Prise KM, Currell FJ, et al: Cell-specific radiosensitization by gold nanoparticles at megavoltage radiation energies. Int J Radiat Oncol Biol Phys 79: 531-539, 2011.

13. Chang MY, Shiau AL, Chen YH, Chang CJ, Chen HH and Wu CL: Increased apoptotic potential and dose-enhancing effect of gold nanoparticles in combination with single-dose clinical electron beams on tumor-bearing mice. Cancer Sci 99: 1479-1484, 2008.

14. Mousavie Anijdan SH, Mahdavi SR, Shirazi A, Zarrinfard MA and Hajati J: Megavoltage X-ray dose enhancement with gold nanoparticles in tumor bearing mice. Int J Mol Cell Med 2: 118-123, 2013.

15. Zhang XD, Wu D, Shen X, Chen J, Sun YM, Liu PX and Liang XJ: Size-dependent radiosensitization of PEG-coated gold nanoparticles for cancer radiation therapy. Biomaterials 33: 6408-6419, 2012.

16. Joh DY, Sun L, Stangl M, Al Zaki A, Murty S, Santoiemma PP, Davis JJ, Baumann BC, Alonso-Basanta M, Bhang D, et al: Selective targeting of brain tumors with gold nanoparticle-induced radiosensitization. PLoS One 8: e62425, 2013.

17. Hainfeld JF, Smilowitz HM, O'Connor MJ, Dilmanian FA and Slatkin DN: Gold nanoparticle imaging and radiotherapy of brain tumors in mice. Nanomedicine (Lond) 8: 1601-1609, 2013.

18. Wang $\mathrm{M}$ and Thanou $\mathrm{M}$ : Targeting nanoparticles to cancer. Pharmacol Res 62: 90-99, 2010.

19. Brun E, Sanche L and Sicard-Roselli C: Parameters governing gold nanoparticle X-ray radiosensitization of DNA in solution. Colloids Surf B Biointerfaces 72: 128-134, 2009.

20. Vecchio G, Galeone A, Brunetti V, Maiorano G, Sabella S, Cingolani R and Pompa PP: Concentration-dependent, size-independent toxicity of citrate capped AuNPs in Drosophila melanogaster. PLoS One 7: e29980, 2012.

21. Choi SY, Jeong S, Jang SH, Park J, Park JH, Ock KS, Lee SY and Joo SW: in vitro toxicity of serum protein-adsorbed citrate-reduced gold nanoparticles in human lung adenocarcinoma cells. Toxicol In Vitro 26: 229-237, 2012.

22. Noël C, Simard JC and Girard D: Gold nanoparticles induce apoptosis, endoplasmic reticulum stress events and cleavage of cytoskeletal proteins in human neutrophils. Toxicol In Vitro 31: 12-22, 2016.

23. Butterworth KT, Coulter JA, Jain S, Forker J, McMahon SJ, Schettino G, Prise KM, Currell FJ and Hirst DG: Evaluation of cytotoxicity and radiation enhancement using $1.9 \mathrm{~nm}$ gold particles: Potential application for cancer therapy. Nanotechnology 21: 295101, 2010 